\title{
MANNED MISSION TO AN ASTEROID
}

\author{
HARVEY HALL \\ NASA, Office of Manned Space Flight
}

If manned missions to the planets are to be considered at some time in the future, as has long been imagined, it appears to be reasonable to explore the feasibility of carrying out a manned mission to an asteroid as an intermediate step between lunar and planetary missions. If study shows that it is possible to conduct such a mission with Apollo-type hardware, and if the answer to the first question is yes, then an asteroid flight should probably be considered while Apollo-type facilities are still in existence. Once these facilities have been dismantled, it may be many years, perhaps a generation, before a new era in space exploration would make such a manned flight possible.

With these thoughts in mind, an analysis was undertaken to determine whether an asteroid mission is practicable. An overriding consideration is the reliability of the life-support system. Life-support systems for Skylab-the final manned mission now being planned after Apollo-are being designed for missions of 56 days. Most planetary round-trip missions that have been studied would take much longer than this to complete; namely, of the same order as the planet period, or a year or so. It would presently be impracticable to design life-support systems for such long missions, approximately 10 times as long as the Skylab mission. Consequently, to be considered as a possibility, an asteroid mission based on the use of Apollo-type hardware would have to be found that could be completed within a couple of months.

To explore this question, it is necessary to know how the difficulty of such a mission would compare with a mission to Mars. The comparison is in terms of the total $\Delta v$ and the total mission time required. For purposes of comparison, a rough approximation of $\Delta v$ needed for two types of missions is given in table I.

The total mission $\Delta v$ provides a sensitive indicator of mission difficulty or cost because once the types of rocket fuel, engines, etc., have been selected,

TABLE 1.-Comparison of Missions

\begin{tabular}{l|c|c}
\hline \multicolumn{1}{c|}{ Mission } & Total $\Delta \nu, \mathrm{km} / \mathrm{s}$ & Mission time \\
\hline Moon (Apollo) & 20 & 1 week \\
Mars & 40 & $1 \mathrm{yr}$ \\
\hline
\end{tabular}


the mass ratio, or ratio of gross takeoff weight to gross weight less fuel, depends exponentially on $\Delta v$. To double $\Delta v$, for example, may increase total takeoff weight by a factor of 7 or more. The total cost may easily increase at even a higher rate, depending on the amount of new technology needed.

Total mission time is also a sensitive index of mission cost in the present state of the art. The reason for this is that the cost of extending the operating time of the life-support and other essential subsystems needed, while maintaining the same level of mission reliability, may be greater than the cost of the original subsystems, even many times greater. For this reason, an asteroid mission that could be completed in 2 or 3 months, with perhaps $2 / 3$ the $\Delta v$ requirements of a Mars mission, might have much to recommend it as an intermediate U.S. mission.

In this paper, an approximate method has been derived for studying the cases that would be worth considering. Generally these are limited to asteroids with the following orbital features:

(1) Perihelion in or near the ecliptic plane

(2) Perihelion within $\sim 0.1 \mathrm{AU}$ of Earth's orbit

(3) Aphelion less than approximately 1.5 or $2 \mathrm{AU}$

(4) Angle between orbital plane and ecliptic plane less than $10^{\circ}$ to $12^{\circ}$

(5) Launch window available at desired time

There are probably many asteroids that meet the first four conditions. Some of them (e.g., Eros) have a launch window within the next 5 to $10 \mathrm{yr}$. But better choices may be available at a later time because not all of the ephemerides are accurately known, and further work done in cataloging and classifying them might reveal a number of interesting new possibilities.

The results of the study show that

(1) Mission times of the order of 3 months or less are feasible.

(2) Asteroid missions needing a total $\Delta v$ of 24 to $30 \mathrm{~km} / \mathrm{s}$, or perhaps somewhat less, exist.

(3) The built-in requirements that exist for all missions create a basic minimum $\Delta v$ that cannot be reduced much below 18 to $21 \mathrm{~km} / \mathrm{s}$ unless a "freak" asteroid that comes very close to Earth can be found.

The more or less irreducible requirements include

(1) Earth escape: $10 \mathrm{~km} / \mathrm{s}$

(2) First retro (or speedup) from Earth: 0.9 to $0.6 \mathrm{~km} / \mathrm{s}$

(3) Correction for radial velocity gained (or lost) during transit: 1.5 to $2 \mathrm{~km} / \mathrm{s}$

(4) Speedup (or slowdown) to asteroid velocity prior to rendezvous and docking: 2 to $10 \mathrm{~km} / \mathrm{s}$

(5) Plane change (if any): 0 to $4.5 \mathrm{~km} / \mathrm{s}$ 
(6) Orbital transfer from asteroid to Earth (equal radial and transverse transit times): $3 \mathrm{~km} / \mathrm{s}$

(7) Final plane change (if any): 0 to $4.5 \mathrm{~km} / \mathrm{s}$

Only the root mean square is needed of items (3) through (7). However, there is a practical minimum for $\Delta v$ as noted above, and a minimum of perhaps 6 to 8 weeks transit time for even the most accessible asteroid.

Eros, for which the next launch window will be in 1975 , is not a favorable case from the viewpoint of total $\Delta v$. The total $\Delta v$ needed in 1975 would be comparable with the amount needed for a Mars mission. This tends to rule out Eros although the total mission could be completed in the favorable time of about 90 days, compared with over a year for Mars.

To establish a lower limit on $\Delta v$ and mission time, a hypothetical asteroid was studied having the following orbital characteristics:

$$
\begin{aligned}
r_{P} & =0.99 r_{E} \\
r_{A} & =1.4 r_{E} \\
\phi & =0
\end{aligned}
$$

where $r_{P}$ is the perihelion distance, $r_{A}$ is the aphelion distance, $r_{E}$ is the Sun-Earth distance, and $\phi$ is the angle between the orbital plane and the ecliptic. Such an asteroid may not exist. Many small asteroids having favorable orbital characteristics have been observed over the years, only to be lost again before their orbits could be established.

The requirements are still of the order of magnitude of 20 to $24 \mathrm{~km} / \mathrm{s}$. The results for this hypothetical asteroid show that mission times as short as 6 or 7 weeks are feasible. 